Patients were seen from both inpatient and outpatient setting in 2017 and mainly from outpatient setting from January to June 2018.

115 patients were enrolled out of 1410 patients in 2017 and 84 patients out of 1434 in 2016. This represented 2.3\% increase in enrolment rate. From January to June 2018, 376 patients were seen and 69 patients enrolled. Average yield from June 2013 to December 2016 was 5.4\%, which was considered low. Patients yield increase from 8.2\% in 2017 to $18.4 \%$ within the first 6 months of 2018. In 2013 to 2016, we only managed to enrol 6 patients per month. The enrolment rate increase to 10 patients per month in 2017 and 12 patients per month in 2018.

There is a significant increase in ACP enrolment rate after shifting our focus from inpatient to outpatient setting. Patients and their caregivers are not ready for ACP conversations during hospitalisation as patients could be too sick to engage in the conversations. They are more willing and receptive to have ACP conversations when they are in outpatient setting. Moving forward, the ACP team will continue to work with primary care providers to offer ACP to patients who need them earlier in their disease trajectory.

\section{P52 OPPORTUNITY TO PRACTICE ADVANCE CARE PLANNING AS REGARDED BY NURSES CARING FOR PEOPLE WITH CHRONIC ILLNESSES IN JAPAN}

${ }^{1} \mathrm{M}$ Yoshida*, ${ }^{1} \mathrm{~K}$ Honjo, ${ }^{1} \mathrm{M}$ Morita, ${ }^{1} \mathrm{~T}$ Tanaka, ${ }^{1} \mathrm{Y}$ Higuchi, ${ }^{1} \mathrm{Y}$ Tonoki, ${ }^{1} \mathrm{C}$ Sakai, ${ }^{1} \mathrm{Y}$ Sumiya, ${ }^{2} \mathrm{~A}$ Tanaka, ${ }^{3} \mathrm{M}$ Nakamura. ${ }^{1} J a p a n e s e$ Red Cross College of Nursing, Tokyo, Japan; ${ }^{2}$ Showa

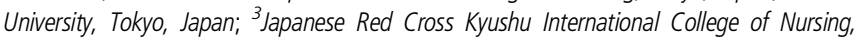
Fukuoka, Japan

\subsection{6/spcare-2019-ACPICONGRESSABS.135}

Purpose As the Japanese population ages and advanced medical care progresses, there is an increasing need to consider ACP for people living with chronic illnesses. Nurses assume an important role in the advancement of ACP. The purpose of this study is to clarify the opportunity to put it into practice as perceived by nurses who are providing nursing care for patients with chronic illnesses.

Methods A quantitative study design was employed using a self-administered questionnaire survey. The questionnaire consisted of one to five Likert scales, and was sent via post to 1,855 addressees including all the Certified Nurse Specialists in Japan engaged in nursing care of people with chronic illnesses. 693 nurses responded.

Results The scores were high for "Setting up a meeting as required (during hospitalization)" (4.50 \pm 0.62$)$, "Setting up a meeting as required (as an outpatient) (4.43 \pm 0.69$)$, "At the time of discharge (including the meeting to discuss treatment details with the patient and personnel involved in their treatment) $(4.17 \pm 0.83)$, and "When providing regular treatment or nursing care (during hospitalization) (4.11 \pm 0.82 ).

Discussion The items raised as an opportunity to practice ACP, namely, "As required" and "When providing regular treatment or nursing care" are due to the characteristics of chronic illnesses, which require a long period of recuperation and carry uncertainty in the illness trajectory.

\section{P53 STUDY PROTOCOL FOR A RANDOMIZED CONTROLLED TRIAL ON THE EFFECTIVENESS OF ADVANCE CARE PLANNING (ACP) IN GENERAL PRACTICE}

J Stevens*, A de Vleminck, P Pype, L Deliens, K Pardon. VUB, Brussels, Belgium

\subsection{6/spcare-2019-ACPICONGRESSABS.136}

Background General practice is an optimal setting for ensuring timely initiation of ACP discussions. A multi-component ACP intervention developed for the general practice setting, aimed at patients with chronic life-limiting illnesses and their general practitioner, has been pilot tested. The aim of this study is to evaluate the effectiveness of this intervention and to evaluate its implementation by means of a process evaluation.

Methods/Design Using a cluster-randomized controlled trial (RCT) (randomization at the practitioner level, $n=53$ practitioners per condition, $n=133$ patients per condition), we will compare the structured ACP communication intervention to usual care, employing baseline measures (T0), and follow-up at 6 months (T1) and 12 months post-baseline (T2). Primary endpoints are quality of communication about end-of-life care and concordance between patients' preferences and received care at the end of life (as reported by the family caregiver if the patient died). Other patient or caregiver-reported outcomes include health-related quality of life, anxiety and depression, quality of end-of-life care, and quality of death and dying. Following the RE-AIM framework, structured diaries for trainers and general practitioners, as well as qualitative interviews with general practitioners, patients and family caregivers are among the measures used for the process evaluation.

Discussion After this Phase III RCT, we will be able to present a well-tested and evaluated ACP intervention that can be implemented in general practice. The results of the process evaluation will provide insight needed to allow adaptation of the intervention for a greater variety of national and international contexts.

\section{P54 HOW PATIENTS AND RELATIVES PERCEIVE THE CONCEPT OF ADVANCE CARE PLANNING: AN INTERVIEW STUDY}

${ }^{1} \mathrm{D}$ van der Smissen* ${ }^{*}{ }^{1} \mathrm{~J}$ Rietjens, ${ }^{2} \mathrm{~S}$ van Dulmen, ${ }^{3} \mathrm{~J}$ van Gemert-Pijnen, ${ }^{1} \mathrm{~A}$ van der Heide, ${ }^{1}$ I Korfage. 'Erasmus University Medical Center, Rotterdam, Netherlands; ${ }^{2}$ NIVEL, Utrecht, Netherlands; ${ }^{3}$ University of Twente, Enschede, Netherlands

\subsection{6/spcare-2019-ACPICONGRESSABS.137}

Background The EAPC consensus concept defines Advance Care Planning (ACP) as enabling persons to identify goals and preferences for future treatments and care, and to discuss, record and review these. However, this concept is defined by professionals. It is unknown how patients and relatives conceptualize ACP, however, this would be useful for (online) information provision on ACP.

Aims To explore what patients and relatives consider important in ACP and how they would search for ACP information on the internet. 
Methods To address our aims, we interviewed 9 patients with chronic diseases and 7 relatives. We used constant comparative analyses to categorize their key elements of ACP.

Results Patients and relatives would use search terms such as 'ACP', 'care/treatment plan', 'disease trajectory', 'advance directive' and patient associations. They would appreciate information on ACP and its importance; how to adapt ACP to individual needs; values and quality of life; diseases; treatment and care options; and communication of preferences. Furthermore, interviewees mentioned additional needs, such as how to get support, explanation of persons involved in ACP, encouragement to think about ACP, information for relatives, and peer support.

Conclusion(s) Key elements of ACP according to patients and relatives are in line with the EAPC consensus concept according to professionals. However, patients and relatives also mentioned additional elements, indicating their concept of ACP may be broader. Including the elements and search terms in ACP information may enhance online findability and may help meeting information needs.

Funding The Netherlands Organisation for Health Research and Development (ZonMw).

\section{P55 NURSES' UNDERSTANDING AND PERSPECTIVES OF ADVANCE CARE PLANNING IN COPD}

C Kelly, N Cornally*. UCC graduate, GALWAY, Ireland

\subsection{6/spcare-2019-ACPICONGRESSABS.138}

Background Advance care planning (ACP) presents as a current issue within an Irish context. Recent publications by The Irish Hospice Foundation include documents 'Think Ahead' and 'Planning for the Future with COPD'. In 2015 The Assisted Decision-Making Capacity Act was published. Currently, the HSE are in the process of publishing professional guidelines for practice in relation to ACP (HSE 2018.) Much of the existing literature relates to physician's views on ACP in COPD. Articles relating to nurse's understanding of ACP entail more towards other patient subgroups such as oncology patients and the older adult.

Method A descriptive qualitative design was used. Eight staff nurses from a respiratory unit were recruited. Semi-structured interviews were audio-recorded. A topic guide from a previous study was adapted.

Results Four categories were identified; the importance of ACP in COPD, facilitators to ACP in COPD, barriers to ACP in COPD and the role of the nurse. Each category was further subcategorised.

Conclusion Nurses identified the importance of ACP in COPD. Benefits included symptom specific factors but also an improvement in the overall quality of care patients could receive. A number of facilitating factors and challenges were identified by the sample in the provision of ACP. Nurses identified themselves as playing an important and broad role in ACP. Future implications were identified for education, research and practice. Recommendations include an increase in education across both undergraduate and postgraduate forums, further research and the appointment of lead nurses in relation to ACP in COPD patients.

\section{P56 ENHANCING THE END OF LIFE (EOL) COMMUNICATION: EVALUATION OF THE 'EOL CARE EDUCATION' FOR NURSES IN ACUTE CARE SETTING}

${ }^{1} \mathrm{M}$ Eto*, ${ }^{2} \mathrm{~K}$ Fujimoto, ${ }^{1} \mathrm{C}$ Koyama, ${ }^{1} \mathrm{~N}$ Ishikawa, ${ }^{1} \mathrm{M}$ Shimada, ${ }^{1} \mathrm{Y}$ Maehara. ${ }^{1}$ Bell-land General Hospital, Sakai, Osaka, Japan; ${ }^{2}$ Hannan City Hospital, Hannan, Japan

\subsection{6/spcare-2019-ACPICONGRESSABS.139}

Background It is critical to discuss with advance cancer patients on their EOL issues to improve the quality for their future care. Japanese nurses, however, are apt to feel uncomfortable to deal with such discussions with these patients because most of them have limited opportunities for the training for EOL communication.

We investigated whether 'EOL care education course' for nurses may help improving their knowledge and skills on EOL care, including communication ability.

Methods We provided 15 nurse participants with the educational course based on the ELNEC Core Curriculum including communication and ethical consideration. They all had more than 2 to 25 years of nursing experience. Efficacy of the course was evaluated by using the End of Life Nursing Education-Japan Core Quiz.

Results The overall correct answer rate before the course was $66 \%$ which was significantly improved to $86 \%$ after the training $(p<0.05)$. The learning effect was obtained about the knowledge and attitude towards the EOL care by taking the course. The participants felt it challenging to deal with ethical facets and patients' spiritual pains. The results, however, showed an increase in the correct answer rates on 'communication skills' from $70 \%$ to $93 \%$, before and after the course, respectively. We believe that the practical role play during the course contributed this improvement.

Conclusions The education course we provided improved knowledge and skills for EOL communication in the nurse participants. We learned that in the future the course needs more attention on the 'ethical consideration' and 'spiritual care'.

\section{P57 COLLABORATIVE ADVANCE CARE PLANNING: IMPROVING PALLIATIVE CARE BY STRUCTURED COMMUNICATIONS ABOUT MATTERS OF LIFE AND DEATH - THE STUDY PROTOCOL}

${ }^{1} \mathrm{~J} \mathrm{Koch}{ }^{*},{ }^{1} \mathrm{M}$ Koch, IJ Riera-Knorrenschild, ${ }^{2} \mathrm{~N}$ Timmesfeld, ${ }^{3} \mathrm{~N}$ Leppin, ${ }^{3} \mathrm{~K}$ Nagelschmidt, ${ }^{1} \mathrm{P}$ Von Blanckenburg. ${ }^{1}$ Philipps-University Marburg, Marburg, Germany; ${ }^{2}$ Ruhr University, Bochum, Germany; ${ }^{3}$ Philipps University, Marburg, Germany

\subsection{6/spcare-2019-ACPICONGRESSABS. 140}

Background Implementation of advance care planning (ACP) is proceeding in the western world and often recognised as a sufficient approach to ensure patients' wishes for end of life (EoL) care. There is evidence that patient related outcomes are improved. However, information about the impact of ACP on quality of life (QoL) in palliative cancer patients is missing.

Methods This randomised controlled trial investigates the efficacy and effectiveness of a collaborative ACP (cACP) intervention in palliative cancer patients and their care givers by comparing three groups: 1. cACP-Intervention; 2. Supportive intervention 3. Treatment as usual. The cACP-intervention 